\title{
Infrared Thermography and DIT of Quadcopter Rotor Blades Using Laser Heating ${ }^{\dagger}$
}

\author{
Raffael Thiessen and Erich Schülein * \\ German Aerospace Center DLR, Institute of Aerodynamics and Flow Technology, Bunsenstr. 10, 37073 \\ Göttingen, Germany; raffael.thiessen@web.de \\ * Correspondence: erich.schuelein@dlr.de \\ † Presented at the 15th International Workshop on Advanced Infrared Technology and Applications \\ (AITA 2019), Florence, Italy, 17-19 September 2019.
}

Published: 25 September 2019

\begin{abstract}
A measuring method to detect wall flow topologies by active infrared thermography was developed. Quadcopter rotor blades were studied in typical hover and forward flight conditions, while using a high-power infrared laser to heat the blade surface. By this, the distance between heat source and measuring object could be significantly increased compared to conventional heat sources. The resulting images show little blurring at high signal-to-noise ratios. In steady-state flow conditions, wall flow topologies could be detected reliably and show good agreement with performed oil-film interferometry measurements. Wind tunnel experiments at forward flight conditions were conducted and indicate unsteady shifts of the transition location.
\end{abstract}

Keywords: thermography; boundary-layer transition; rotor blades; oil-film interferometry; laser heating; differential infrared thermography

\section{Introduction}

Infrared (IR) thermography is widely used and offers a non-intrusive and comprehensive method for transition detection and flow-topology visualization. This method relies on the increase in heat transfer rate between the flow and the surface occurring due to boundary-layer transition from laminar to turbulent state. While adiabatic recovery temperatures are lower in laminar flows than in turbulent flows, the resulting temperature differences in subsonic flow regimes are typically not sufficient for detailed thermographic measurements. In order to increase differences in temperature between the areas with laminar and turbulent flows, external and/or internal heating of the testing surface is often unavoidable. External heat sources, e.g., halogen lamps [1,2] or recently flash lamps [3] offer high optical power outputs, but need to be positioned close to the measurement object to compensate for high divergence angles of the light cone. This can limit its usability in large industrial wind-tunnel facilities, as the inflow may not be obstructed or altered by the measurement equipment. Internal heating of testing surfaces, e.g., by resistance heating [4], overcomes these shortcomings. Nevertheless, accommodating an internal heat source into the test model can be difficult.

With the use of modern high-power industrial IR lasers, high heat-flux densities can be achieved while maintaining a distance of several meters between the heat source and the model. Further advantage is the compact laser head which can be used as external heating source even inside the test section of large wind tunnels without significantly changing the incoming flow. In this study, laser heating is applied for thermography measurements and a corresponding measurement method is developed. This study aims to demonstrate the applicability of this method to detect flow topologies of fast-rotating quadcopter rotors, as well as to reveal the limitations of this technique. To 
determine, whether a detection of unsteady changes in flow topology is possible, differential infrared thermography was additionally performed in a wind tunnel to simulate flow conditions in forward flight. Phase shifts in laminar-turbulent transition expected between advancing and retreating blades were determined. Even state-of-the-art high-speed IR cameras reach their limits when it comes to very short exposure times accompanied by the increased noise and diminished temperature resolution. Phase-averaging of images, taken as a sequence via a once-per-revolution trigger signal, was used in the present work to enhance the image quality, while maintaining short exposure times.

\section{Materials and Methods}

Thermographic measurements were performed on four differently sized and shaped quadcopter rotors (Mejzlik), made from carbon fiber reinforced polymer (CFRP). They are referred to as e.g., R18x6, representing a diameter of $18^{\prime \prime}$ and a pitch of $6 "$. For increased emissivity, a black matt coating was applied to the rotor surfaces. An industrial infrared diode laser (Jenoptik JOLD-1250-CABN-25A) with a wavelength of $938 \mathrm{~nm}$, was used to heat the suction side surface of the rotor blade. Compared to conventional heat sources like halogen lamps, a laser of this type offers very high heat fluxes over large distances. For this study, the distance between laser and measurement object was $5 \mathrm{~m}$. A high-speed infrared camera (IRCAM EQUUS 327k L) with a resolution of $640 \times 512$ pixels and a spectral range of $7.7<\lambda<9.4 \mu \mathrm{m}$, was operated in triggered mode. By the large spectral distance to the laser's wavelength, a direct detection of reflections is ruled out. A model aircraft motor (D-POWER AL 50-04) was used to drive the rotors. Every set of blades was studied at three rotational frequencies $f_{1}=50 \mathrm{~Hz}, f_{2}=80 \mathrm{~Hz}$ and $f_{3}=100 \mathrm{~Hz}$. With an exposure time of $20 \mu \mathrm{s}$, a good tradeoff between camera noise and motion blur could be achieved.

Due to limitations of the laser's power supply, minimal pulse lengths ranged around $2 \mathrm{ms,}$ which is significantly higher than transit time of a point at the blade tip through the laser beam at the given rotational frequencies. Hence, the rotor was heated during the whole transit time. To help compensate radially varying transit times and to reduce the thermal load on the rotor hub, a triangular-shaped aperture was introduced between laser and rotor, leading to a heating sector of $14^{\circ}$. In steady-state experiments the hub is little cooled by the flow, leading to an accumulation of heat and possible damage by overheating. Optical power output of the laser was set to $700 \mathrm{~W}$, in order to rule out overheating at the hub. Furthermore, surface heating should be kept as low as possible, as too high temperatures might impact the boundary layer [4].

A trigger signal was generated by the blade passing through a light barrier for every revolution of the rotor. This signal was used to activate the laser and trigger the infrared camera shortly after. Since camera noise limits the achievable temperature resolution at low exposure times, a phase-averaging method to reduce noise and increase image quality was applied. While rotating with constant frequency, the rotor blade was periodically heated. After reaching a thermal equilibrium state, a series of 100 phase-locked images of the blade were recorded. Those images were then averaged pixel by pixel and one phase-averaged image was created in this way. Adiabatic surface temperatures increase from hub to tip due to radially increasing Mach numbers. By the subtraction of a reference image of the rotating blade with its equilibrium adiabatic temperature distribution from an image of the pulse-heated blade, this factor is removed from the images. Especially in flow regions near the blade tip, the effects of viscous heating can outshine contrasts due to flow topology. Furthermore, bad pixels were identified from the image by comparing each pixel's temperature value to its eight direct neighbors, and substituting those, that show a deviation of more than $5 \%$ from the median value of those neighbors, by the median value. Subsequently a temperature correction was applied: At different radii, sampling points of the brightest area at each radius, i.e. temperature maxima, were manually selected and a maximum temperature function was interpolated. Temperature values of all pixels were then scaled to the local maximum, in order to account for non-uniform heating of the blade. Image quality could be improved significantly.

Aiming to validate the results from thermographic measurements, oil-film interferometry measurements were performed in order to visualize flow topologies using an independent method 
[5]. The oil film is subjected to both shear stresses imposed by the flow and induced centrifugal forces resulting from the rotational motion of the blade. An analysis of the oil-flow deflection angles provides information about the boundary-layer state. As local wall shear stresses increase significantly during laminar-turbulent transition, a sudden change in deflection angles is expected at the transition line. For each experiment, a thin layer of silicon oil was applied to the rotor blade. The rotor was then spun at constant rotational frequency, until the oil distribution showed comprehensive interference patterns, and an image was taken. Since the internal structure of the uncoated CFRP-blades is visible and overlying the interference patterns, a reference image of the blade without oil applied was recorded and subtracted from the measurement image.

In forward flight, the flow topology is highly dependent on the azimuthal position of the blade, as the rotor's rotary motion and forward motion of the aircraft add together. The wall flow topology constantly changes, with the largest difference within one revolution expected to be between advancing and retreating blade, orthogonal to the direction of flight. Differential infrared thermography (DIT) [1] aims to detect unsteady changes in flow topology by subtracting two IR images recorded in short succession, in order to expose any short-time changes in flow topology, e.g., the detection of shifts in laminar-turbulent transition during one revolution. Forward flight conditions were recreated in a wind tunnel with a freestream velocity of $\boldsymbol{v}_{\infty}=15 \mathrm{~m} / \mathrm{s}$, orthogonal to the rotational axis of the rotor. Thermographic measurements at $f_{3}=100 \mathrm{~Hz}$ were performed on advancing and retreating blades.

\section{Results and Analysis}

To gain a basic understanding of the flow topology and to help with the interpretation of the IR images, oil-film interferometry measurements are analyzed. Wall streamlines of the oil flow on the rotor surface are visible in the interference pictures. Because of the significantly different material properties of oil and air, visible oil streamlines do not necessarily represent air streamlines. Flow direction is from top to bottom in all pictures shown. Figure 1 shows the processed interference picture of rotor R17x5.7, as well as a derived sketch of the oil-flow topology.

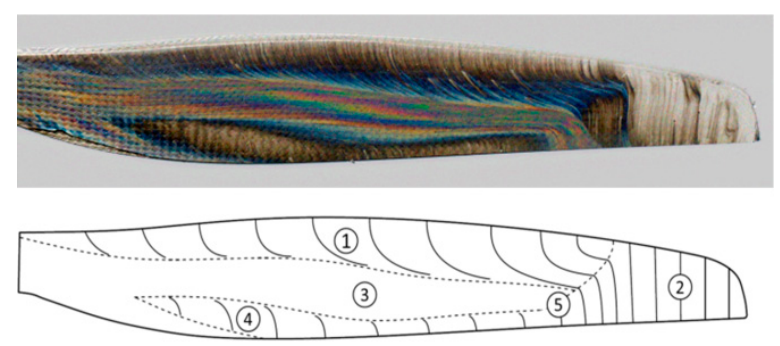

Figure 1. (top) Interference image; (bottom) derived oil-flow topology, rotor R17x5.7, $f_{3}=100 \mathrm{~Hz}$.

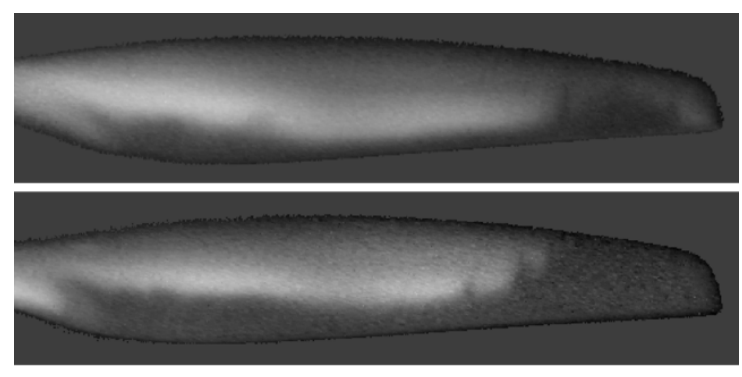

Figure 2. Processed infrared images of rotor R18x6: (top) at $f_{1}=50 \mathrm{~Hz}$; (bottom) at $f_{3}=100 \mathrm{~Hz}$.

For most of the span, the flow starts laminar from the leading edge. Only in an area near the blade tip (2), turbulent flow extends nearly from leading edge to trailing edge. The border between laminar and turbulent flow areas is marked by a sudden deviation of the oil-flow towards the trailing edge due to a leap in wall shear stress. Downstream of the laminar area, the flow experiences 
a positive pressure gradient and is decelerated until it fully separates from the surface. While separated (3), laminar-turbulent transition of the shear layer occurs and the more energetic turbulent flow reattaches again further downstream (4). In the interference image, the laminar separation bubble is characterized by nearly radial wall streamlines. Since the flow is separated, the oil flow is only subjected to centrifugal forces. Deviations from an undisturbed radial flow inside the separation bubble can be explained by the rotor's geometry. The oil is pushed off the surface when it reaches the turbulent flow area (5). An increase in frequency leads to an increase in inflow Mach number for a given point at the leading edge and the area of turbulent flow at the blade tip is enlarged. In addition, the chordwise length of the separation bubble decreases as the turbulent flow reattaches faster. Distinct structures on the rotor surface can be identified in the IR images. Subtracted IR images of the Rotor R18x6 for $f_{1}$ and $f_{3}$ are displayed in Figure 2. From the leading edge, brightness gradually increases in streamwise direction, which can be attributed to a rather uniform buildup of the boundary layer. At the point where the wall shear stress is decreased to zero, the flow separates. The turbulent flow reattaches behind the separation bubble, which is marked by a sudden change from brighter to darker colors. Higher brightness indicates higher temperature differences between laser-heated and reference image, corresponding to laminar or separated flow areas, as surface cooling by the flow in those areas is lower than in turbulent areas.

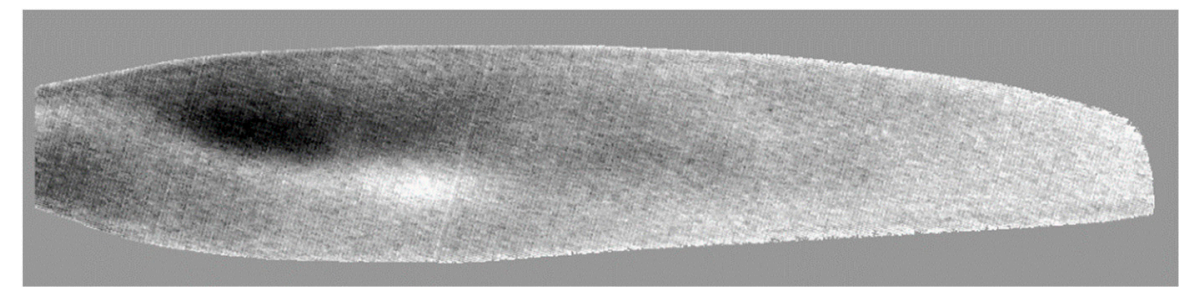

Figure 1. DIT Image of rotor R18x6 at $f_{3}=100 \mathrm{~Hz}$

A DIT image of the rotor R18x6 at $f_{3}=100 \mathrm{~Hz}$ with enhanced contrast is shown in Figure 3. Since the image of the advancing blade is subtracted from the image of the retreating blade, brighter colors represent higher temperatures on the retreating blade; dark colors vice versa. A plausible shift in laminar-turbulent transition is indicated by the DIT images for a part of the blade radius near the hub. A streamwise change from darker to brighter colors could be explained by a slight shift within one revolution: At a given radius the advancing blade experiences higher Mach numbers, which may lead to an earlier separation, as well as to faster transition and reattachment. A significant positive temperature difference, as seen in brighter areas, may be due to a further extent of the separation bubble at the retreating blade, while the already turbulent flow at the advancing blade would lead to decreased surface temperatures. Although these DIT measurements indicate some short-time changes in transition onset, no flow structures are visible for the outer half of the blade. This may be attributed to the fact that relative differences in inflow Mach numbers between advancing and retreating blades are higher towards the hub. The achievable temperature differences with the given heat input are not sufficient for detection by the infrared camera. Further investigations into this technique may be necessary. Because of highly unsteady flow conditions in forward flight, it is not possible to validate the DIT measurements by oil-film interferometry.

\section{Conclusions}

IR thermography measurements have been performed on spinning quadcopter rotor blades in hover and at forward flight conditions. A high-power infrared laser has been used to heat the blade surface. Thereby the distance between heat source and measurement object could be significantly increased compared to conventional external heat sources, allowing for thermography to be applied in large wind tunnels or where obstructions of the flow would be otherwise inevitable. Signal-to-noise ratio could be drastically increased by phase-averaging. It could be shown, that an industrial infrared laser can be used to detect steady-state flow topologies at high rotational frequencies reliably and with high image quality. Furthermore, results could be validated by oil-film 
interferometry measurements that were performed on the same rotor blades. The minimal pulse duration was limited by the laser's power supply. With rather long laser pulses, thermal load on the rotor hub was high and limited the achievable heat flux density.

DIT results of forward flight wind tunnel experiments indicate that some unsteady changes in flow topology can be detected: Shifts in laminar-turbulent transition within a single revolution can be partially visualized by subtracting phase-averaged images of advancing and retreating blade. Nevertheless, further work has to be done in order to further improve detectability of unsteady phenomena over the whole extent of the blade. Higher heat flux densities would be a possibility to increase temperature differences, e.g., by increasing optical laser power or using multiple lasers.

\section{Abbreviations}

The following abbreviations are used in this paper:

IR infrared

DIT differential infrared thermography

CFRP carbon fiber reinforced polymer

Author Contributions: conceptualization, E.S.; methodology, R.T. and E.S.; software, R.T. and E.S.; formal analysis, R.T.; investigation, R.T. and E.S.; writing-original draft preparation, R.T.; writing-review and editing, R.T. and E.S.; supervision, E.S.; project administration, E.S.

Funding: This research received no external funding.

Acknowledgments: The authors thank Sebastian Weiss, Jens Lunte and Hartmut Haselmeyer from DLR Göttingen for their assistance in executing the experiments.

Conflicts of Interest: The authors declare no conflict of interest.

\section{References}

1. Richter, K.; Schülein, E. Boundary-layer transition measurements on hovering helicopter rotors by infrared thermography. Exp. Fluids 2014, 55, 1-13.

2. Raffel, M.; Merz, C.B. Differential Infrared Thermography for Unsteady Boundary-Layer Transition Measurements. AIAA J. 2014, 52, 2090-2093.

3. Von Hoesslin, S.; Stadlbauer, M.; Gruendmayer, J.; Kähler, C.J. Temperature decline thermography for laminar-turbulent transition detection in aerodynamics. Exp. Fluids 2018, 58, 129.

4. Simon, B.; Filius, A.; Tropea, C.; Grundmann, S. IR thermography for dynamic detection of laminar-turbulent transition. Exp. Fluids 2016, 57, 93.

5. Schülein, E. Optical method for skin-friction measurements on fast-rotating blades. Exp. Fluids 2014, 55, 1672. 